\title{
The risk and control of Salmonella outbreaks in calf-raising operations: a mathematical modeling approach
}

\author{
Cristina LANZAS*, Lorin D. WARNICK, Renata IVANEK, Patrick AYSCUE, \\ Daryl V. NYDAM, Yrjo T. GRÖHN \\ Department of Population Medicine and Diagnostic Sciences, College of Veterinary Medicine, \\ Cornell University, Ithaca, NY 14853, USA
}

(Received 20 April 2008; accepted 3 September 2008)

\begin{abstract}
Salmonellosis in calves has economic and welfare implications, and serves as a potential source of human infections. Our objectives were to assess the risk of Salmonella spread following its introduction into a herd of pre-weaned calves and to evaluate the efficacy of control strategies to prevent and control outbreaks. To meet these objectives, we developed a model of Salmonella transmission within a pre-weaned group of calves based on a well documented outbreak of salmonellosis in a calf-raising operation and other literature. Intervention scenarios were evaluated in both deterministic and stochastic versions of the model. While the basic reproduction number $\left(R_{0}\right)$ was estimated to be 2.4 , simulation analysis showed that more than $60 \%$ of the invasions failed after the introduction of a single index case. With repeated introduction of index cases, the probability of Salmonella spread was close to 1, and the tested control strategies were insufficient to prevent transmission within the group. The most effective strategies to control ongoing outbreaks were to completely close the rearing operation to incoming calves, to increase the proportion of admitted calves that were immunized (>75\%), and to assign personnel and equipment to groups of calves.
\end{abstract}

calf / Salmonella / transmission model / control

\section{INTRODUCTION}

Salmonella enterica colonizes the gastrointestinal tract of a wide range of hosts [22]. In cattle, Salmonella infection results in both asymptomatic carriage and clinical salmonellosis [22]. Infected cattle are a potential source of human infections through foodborne, direct animal contact and environmental exposures. Although cattle of all ages can be infected with Salmonella, serious infections and high mortality most often occur in calves younger than 10 weeks old [17]. Salmonella outbreaks in calves are serious economic and welfare concerns due to losses from mortality, treatment costs and poor performance. Salmonella outbreaks

*Corresponding author: c1272@ cornell.edu are also a potential source of antimicrobial resistance; an increased resistance to ceftrioxane has been reported during the course of an outbreak in dairy calves ${ }^{1}$.

Calves in raising operations are at high risk of suffering salmonellosis [15]. In raising operations the continual admission of new calves from mixed sources and the highdensity confinement of a large number of susceptible animals favor the transmission of Salmonella. As a result, infection in a limited number of calves can spread very rapidly through the herd. Salmonella transmits predominantly by a fecal-oral cycle [22]. Direct

\footnotetext{
${ }^{1}$ Ray K.A., Epidemiology of antimicrobial resistant Salmonella on dairy farms in the Northeast and Midwest USA, Doctoral thesis, Cornell University, Ithaca, NY, USA, 2007.
} 
transmission occurs when infected animals are housed with susceptible individuals in conditions that favor the immediate transfer of Salmonella from animal to animal. However, Salmonella also survives very well in the environment, which facilitates its indirect transmission through contaminated fomites and mechanical vectors. In individually penned animals, this cross-infection (indirect transmission) between infected animals and contaminated fomites and vectors represents an important route of transmission as suggested by the analysis of the spatial and temporal patterns of Salmonella excretion by calves penned individually [8].

Combined intervention strategies are generally applied during an outbreak. Isolation of sick animals and establishment of strict hygienic procedures are among the most common measures recommended [3]. However, the approach of combining several control measures makes it difficult to assess the efficacy of each measure. Mathematical models of infection transmission provide a framework in which the effect of interventions can be tested, providing specific management guidance under a wide range of situations [1].

Our objectives were to assess the risk of Salmonella spread following introduction in calf-rearing operations and to evaluate the effectiveness of control interventions in order to: (1) control an ongoing Salmonella outbreak and (2) prevent Salmonella outbreaks in operations that are free of Salmonella. For that purpose, we developed a mathematical model of indirect transmission of Salmonella in a calf-raising operation. We used literature and a well documented outbreak in a commercial calf raising unit to obtain the management and transmission parameters [6]. Scenarios simulating different intervention strategies were then evaluated.

\section{MATERIALS AND METHODS}

\subsection{Outbreak description}

The outbreak has been previously described in Gardner et al. [6]. Briefly, in the Fall of 2001, a large commercial dairy calf and heifer raising operation suffered an outbreak of acute neonatal diarrhea. Multidrug-resistant Salmonella serotype Newport was isolated from clinically affected calves and environmental sites. The pre-weaning calves were housed in three identical barns. Each barn had 4 rows with 12 individual pens in each row. Barns were filled as calves arrived, and calves in two adjacent rows facing each other were weaned simultaneously at approximately 5 weeks of age. The index case was reported on August 19, 2001, a three-day-old calf. Over the following 2 weeks, 11 calves housed in the same barn as the index case developed salmonellosis. Although the first cases were limited to one of the barns, cases were reported soon in all 3 barns. During the outbreak, the admission of the calves from the farm where the index case originated was delayed to one week of age and calves were housed in hutches $10 \mathrm{~m}$ away from the barns. By the end of October, the control measures were intensified; calves with serum total protein lower than $5 \mathrm{~g} / \mathrm{dL}$ were rejected and management protocols were revised to reduce Salmonella spread through personnel movement. The new management protocol included the discontinuity of the footbaths (footbaths tested positive for Salmonella), the use of separate boots and coveralls for each barn, and the restriction of the entrance of equipment to the barns. The outbreak lasted until approximately the end of December. After December 21, 2001, no new clinical cases appeared. Overall, the outbreak lasted 124 days, and resulted in 99 clinical cases and 36 deaths.

\subsection{Transmission model}

In our model, calves ('host population') contaminated the workers/equipment (defined in the model as 'vector population'), and contaminated vectors then infected calves completing the transmission cycle (Fig. 1). The model is an SIR model for the calf population coupled with an uncontaminated/contaminated model for the vector population. The model included the following assumptions: (i) calves cannot infect other calves through direct physical contact; (ii) in terms of probability of contact, calves represented a single homogenous population. Although in the outbreak study, calves were initially allocated in three different pre-weaning facilities, the same workers and equipment were used for all three barns; (iii) only Salmonella-induced deaths were considered; (iv) because the time spent in the pre-weaning 
barns was shorter than the duration of the immunity, recovered animals could not become susceptible to Salmonella infection again.

The deterministic model is represented by the following differential equations,

$$
\begin{aligned}
\frac{d S}{d t}= & \delta(1-\phi-\psi)\left(1-\frac{N}{K}\right)-c p_{i} \frac{V^{+}}{V} S \\
& -\varphi(t) q_{S} W \\
\frac{d I}{d t}= & \delta \phi\left(1-\frac{N}{K}\right)+c p_{i} \frac{V^{+}}{V} S-(m+\gamma) I \\
& -\varphi(t) q_{I} W \\
\frac{d R}{d t}= & \delta \psi\left(1-\frac{N}{K}\right)+\gamma I-\varphi(t) q_{R} W \\
\frac{d V^{-}}{d t}= & \alpha V^{+}-c p_{c} \frac{I}{N} V^{-} \\
\frac{d V^{+}}{d t}= & -\frac{d V^{-}}{d t}
\end{aligned}
$$

where the state variables $S, I, R$, and $N$ represent the number of susceptible, infectious, recovered, and total animal population, respectively. $V^{-}$and $V^{+}$represent the number of vectors free of Salmonella and carrying Salmonella, respectively; $V$ represents the overall vector population (Fig. 1). The term $\delta$ is the admission rate of calves (animal $\times$ day $^{-1}$ ). The admission rate is adjusted based on the ratio between the number of animals present in the facilities at a given time $(N)$ and the maximum capacity of the facilities $(K)$. A proportion of the admitted animals can be infected $(\phi)$ or can be immunized $(\psi)$.

The term $W$ is the weaning rate (animal $x$ day $^{-1}$ ). Weaning takes places at discrete-times. $\varphi(t)$ takes a value of 1 when weaning takes places every $f$ days, producing a pulse of amplitude $q W$ (i.e, number of weaned animals), where $q_{S}$, $q_{I}$, and $q_{R}$ represent the proportion of $S, I$, and $R$ at a given time, otherwise $\varphi(t)$ takes a value of 0 (i.e, no weaning takes place). Parameter $\alpha$ is the decontamination rate $\left(\mathrm{day}^{-1}\right), c$ is the contact rate per animal per vector $\left(\right.$ day $\left.^{-1}\right), \gamma$ is the recovery rate $\left(\mathrm{day}^{-1}\right), m$ is the Salmonellainduced mortality rate $\left(\right.$ day $\left.^{-1}\right), p_{i}$ is the proportion of contacts between contaminated vectors and susceptible animals leading to animal infection, $p_{c}$ is the proportion of contacts between infected animals and uncontaminated vectors leading to contaminated vectors.

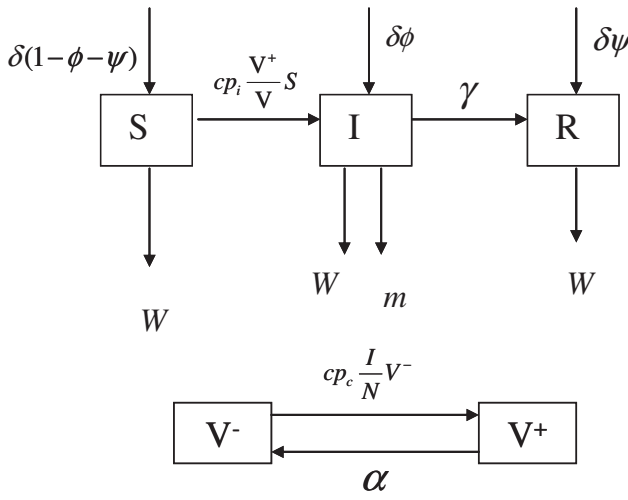

Figure 1. Flow diagram representation of the model.

In small populations, demographic stochasticity (i.e., fluctuations that arise from the random nature of events at the level of the individual) can have a large effect on infection dynamics. Therefore, we also simulated and analyzed the stochastic analog of the deterministic model. In the stochastic version, the random events such as admission, infection, and death were modeled explicitly using the direct method of Gillespie [7].

For the baseline scenario (i.e., scenario without interventions), most of the management and transmission-related parameters were obtained from the documented Salmonella outbreak (Sect. 2.1. and Tab. I). Figure 2 (Panel $a$ ) displays the epidemic curve of clinical salmonellosis. In particular, the early phase of the outbreak (until day 92) was used to estimate the model parameters. This was because during that phase the management protocol remained unchanged compared to the protocol in place before the outbreak and additional control strategies were minimal.

\subsection{Basic reproduction ratio}

The basic reproduction number $\left(R_{0}\right)$ is the threshold quantity that determines the potential of an infectious agent to start an outbreak. We used the next generation matrix approach to derive the $R_{0}$ expression for the model [19]. The next generation matrix $(\mathrm{G})$ is (assuming $\varphi(t)=0$ ),

$$
G=\left(\begin{array}{cc}
0 & \frac{c p_{i}}{\alpha} \\
\frac{c p_{c}}{(m+\gamma)} & 0
\end{array}\right)
$$

(page number not for citation purpose) Page 3 of 13 
Table I. Model parameters used for the baseline scenario.

\begin{tabular}{|c|c|c|}
\hline Symbol & Definition & Value \\
\hline$\alpha$ & Decontamination rate $\left(\right.$ day $\left.^{-1}\right)$ & $0.051^{\mathrm{a}}$ \\
\hline$\beta_{c}$ & Coefficient of contamination $\left(\right.$ day $\left.^{-1}\right)$ & $0.177^{\mathrm{b}}$ \\
\hline$\beta_{i}$ & Coefficient of transmission of infection $\left(\right.$ day $\left.^{-1}\right)$ & $0.177^{\mathrm{b}}$ \\
\hline$\delta$ & Admission rate $\left(\right.$ animal $\times$ day $\left.^{-1}\right)$ & $2^{\mathrm{c}}$ \\
\hline$f$ & Interval between weaning events (day) & $38^{\mathrm{c}}$ \\
\hline$\gamma$ & Recovery rate $\left(\right.$ day $\left.^{-1}\right)$ & $0.062^{\mathrm{d}}$ \\
\hline$K$ & Maximum capacity (animal) & $144^{\mathrm{c}}$ \\
\hline$m$ & Salmonella-induced mortality rate $\left(\mathrm{day}^{-1}\right)$ & $0.047^{\mathrm{e}}$ \\
\hline$\phi$ & Prevalence at admission (dmls) & $0.035^{\mathrm{f}}$ \\
\hline$\psi$ & Proportion of immunized animals at admission (dmls) & $0^{\mathrm{g}}$ \\
\hline$W$ & Weaning rate $\left(\right.$ animal $\times$ day $\left.^{-1}\right)$ & $24^{\mathrm{c}}$ \\
\hline
\end{tabular}

${ }^{a}$ In the absence of interventions, $\alpha$ was based on the survival rate of multidrug-resistant Salmonella Newport in dairy manure [24].

b To obtain transmission parameters from the outbreak data, contact rate $(c)$ and the probability of infection $\left(p_{i}\right)$ or contamination $\left(p_{c}\right)$ were combined into $\beta_{c}$ and $\beta_{i}$ respectively. In addition they were assumed equal and were derived from the intrinsic growth rate of the early phase of the epidemic.

${ }^{\mathrm{c}}$ Demographic parameters were based on the management and capacity of the raising unit.

d The recovery rate was estimated from the available Salmonella faecal shedding data in calves during two outbreaks of $S$. Typhimurium ${ }^{1}$.

${ }^{\mathrm{e}}$ Mortality rate was calculated as the number of deaths divided by the number of days at risk of death times the number of calves at risk, where calves at risk were defined as the animals with salmonellosis and days at risk of death were the mean duration of the infectious period.

${ }^{\mathrm{f}}$ Prevalence at admission was based on the outbreak data [6]. As part of the outbreak investigation, calves were tested in the source farm that was suspected to be the source of the initial Salmonella cases. The ratio between the number of calves admitted from the source farm positive to Salmonella and the total number of admissions was used as baseline value.

g Assumed value.

where the element $g_{12}$ can be interpreted as the expected number of cases in animals produced by one contaminated vector in a susceptible population during the expected time that the vector remains contaminated. The element $\mathrm{g}_{21}$ can be interpreted as the expected number of contaminated vectors produced by one infected animal during its expected infectious period. The basic reproduction number is the dominant eigenvalue of the matrix $\mathrm{G}$,

$$
R_{0}=\sqrt{\frac{c^{2} p_{i} p_{c}}{(m+\gamma) \alpha}}
$$

Here, the values of contact rate (c) and the probabilities of infection $\left(p_{i}\right)$ and contamination $\left(p_{c}\right)$ are unknown. Therefore, we combined them into $\beta_{c}$ and $\beta_{i}$, where $\beta_{c}=c p_{c}$ and $\beta_{i}=c p_{i}$, respectively.

The $R_{0}$ for the case study was estimated from the initial growth rate $(\lambda)$ of the epidemic. During the early phase of the epidemic, the number of cases grows exponentially. The initial growth rate ( $\lambda$ ) can be estimated by fitting the log of the cumulative number of cases in the early epidemic phase to a linear function (Fig. 2, Panel $b$ ). The slope of the linear function is $\lambda$. Mathematically, $\lambda$ is the dominant eigenvalue of the Jacobian matrix evaluated at disease-free equilibrium of the assumed model, and can be obtained by solving the characteristic equation,

$$
\lambda^{2}-(m+\gamma+\alpha) \lambda+(m+\gamma) \alpha-c^{2} p_{i} p_{c} .
$$

By combining both $R_{0}$ and $\lambda$ equations, both quantities can be expressed in terms of model parameters, and related to each other as follows,

$$
R_{0}=\sqrt{1+\frac{\lambda^{2}+(m+\gamma+\alpha) \lambda}{(m+\gamma) \alpha}}
$$

The $R_{0}$ was then used to estimate $\beta_{c}$ and $\beta_{i}$ by assuming that coefficients of transmission were 

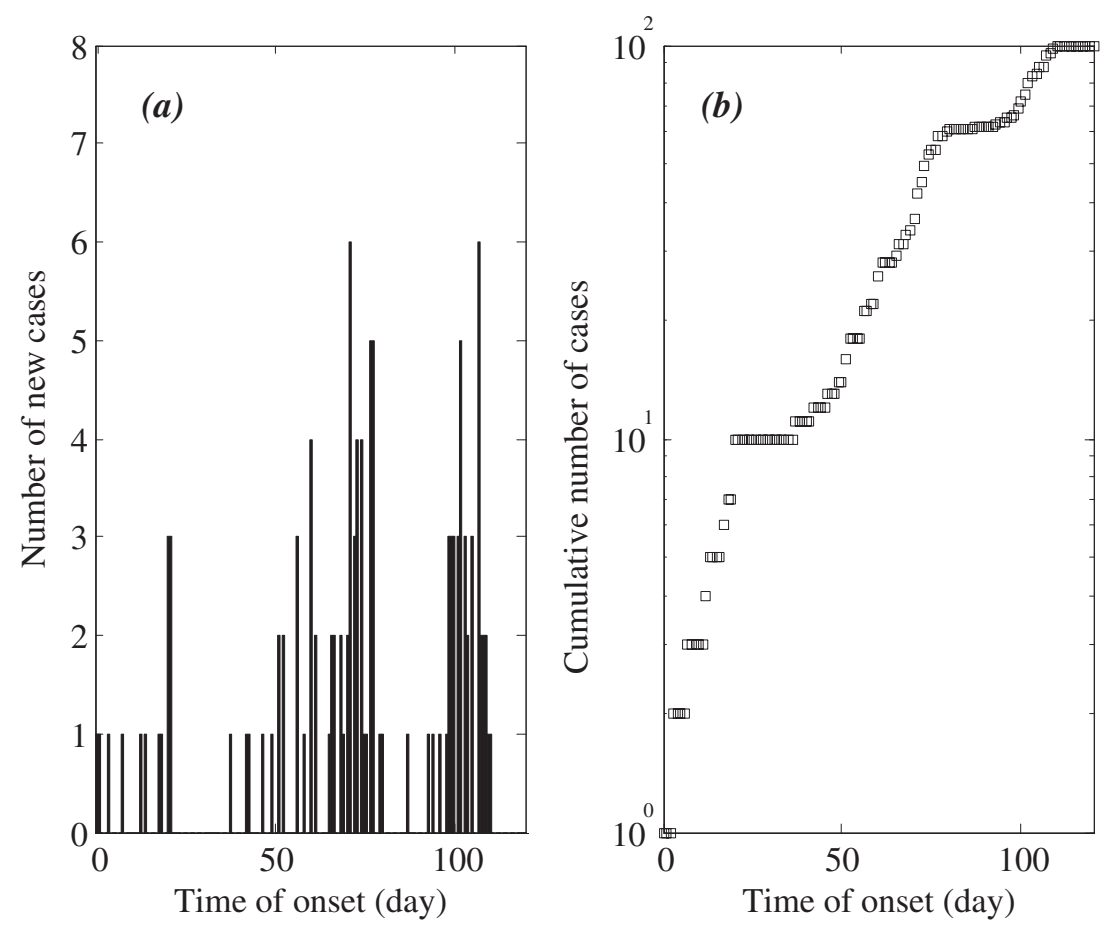

Figure 2. (a) Epidemic curve of clinical salmonellosis for the outbreak described in [6]. (b) Cumulative number of cases during the epidemics (log-lin scale). The estimated initial growth rate of the epidemics was 0.101 with a $95 \%$ confidence interval of $(0.088,0.114)$.

equal. We performed a sensitivity analysis to test this assumption.

\subsection{Risk of Salmonella spread following introduction}

In order to assess the risk of Salmonella spread following introduction into a naïve operation and its consequences, in addition to estimating $R_{0}$, we evaluated the stochastic version of the model. Two types of scenarios were run. In the first set of simulations, we studied the effect that the admission prevalence had on the transmission dynamics. For a given admission prevalence, the number of simulations (from a total of 10000) in which the introduction of infected animals resulted in: (1) no transmission event (i.e., an admitted positive animal did not transmit infection to a single susceptible animal), (2) sporadic transmission events (less than 20 cases), and (3) greater than 20 transmission events a year (excluding the introduced index cases). The cut off of 20 cases per year was chosen as an example of a severe outbreak.

In the second set of simulations, we evaluated the effect of introducing more than one index case at once; this event is most likely to occur when a cluster of animals from a source farm infected with Salmonella are accepted. Simulations were run until outbreaks were extinct; the number of cases and duration of the outbreak were recorded.

\subsection{Intervention strategies to control ongoing Salmonella outbreaks}

Intervention strategies can be classified based on the class (animals or vectors) and on the control point (e.g., reducing the number of susceptibles of the target class or reducing transmission) they target. Although one single control measure may target more than one control point (e.g. vaccination increases the number of animals in the recovered class, but also it may reduce infectiousness), in 
Table II. List of simulated interventions scenarios.

\begin{tabular}{lll}
\hline Control measure & New value & Abbreviation $^{\text {a }}$ \\
\hline Reduction of the admission rate by 50\% & $\delta=1$ & Adm50 \\
Complete closure of the facilities & $\delta=0$ & Adm100 \\
Admission of immunized calves (high coverage) & $\psi=0.75$ & Imm75 \\
Admission of immunized calves (medium coverage) & $\psi=0.37$ & Imm37 \\
Admission of immunized calves (low coverage) & $\psi=0.20$ & Imm20 \\
Reduction of Salmonella shedding by antibiotic treatment (30\% reduction) & $\gamma=0.09$ & Shed30 \\
Reduction of Salmonella shedding by antibiotic treatment (60\% reduction) & $\gamma=0.16$ & Shed60 \\
Reduction of contact rate by 33\% & $\beta_{c}=\beta_{i}=0.128$ & Cont33 \\
Reduction of contact rate by 66\% & $\beta_{c}=\beta_{i}=0.059$ & Cont66 \\
Hygiene measures, decreased environmental Salmonella survival by 33\% & $\alpha=0.076$ & Hyg33 \\
Hygiene measures, decreased environmental Salmonella survival by 66\% & $\alpha=0.153$ & Hyg66 \\
\hline
\end{tabular}

a Abbreviations consists of a three-letter abbreviation for the control measure and the percentage of reduction or increase of the parameter value respective to its baseline value.

order to test the intervention strategies in the model, each intervention was linked to a single parameter (a simplifying assumption). The following control measures were considered,

(i) The continual admission of new susceptible calves can fuel the outbreak, increasing the duration and final size of the outbreak. The effect of reducing admission rate by half $(\delta=1)$ and the complete closure of the facilities $(\delta=0)$ were tested.

(ii) The ingestion of colostrum from vaccinated cows may provide passive protection against salmonellosis in calves. In one study assessing the efficacy of a modified live Salmonella vaccine for adult cattle on a commercial dairy, the frequency of Salmonella fecal shedding by calves that received colostrum from cows vaccinated with the live vaccine was lower than the control group [10]. We evaluated the effect of vaccinating the dams by increasing the proportion of immunized calves that were admitted $(\psi)$. In the default scenario, the proportion of admitted calves that were immune was assumed to be 0 . In addition, three scenarios were investigated. In the best case scenario, all cows were vaccinated and colostrum uptake efficacy was high $(\psi=0.75)$. In the other two scenarios, more conservative vaccine coverage was assessed $(\psi=$ 0.37 and 0.20 ).

(iii) Ceftiofur administered at an extralabel dose reduced fecal shedding of Salmonella in neonatal calves [5]. The time that calves shed Salmonella concentrations higher than the infective dose $\left(10^{5} \mathrm{CFU}\right)$ [14] was reduced by a $60 \%$ in the treated group [5]. Reductions of the duration of Salmonella fecal shedding by $30 \%(\gamma=0.09)$ and $60 \%(\gamma=0.16)$ were tested.

Page 6 of 13 (page number not for citation purpose) (iv) Contact between animals and vectors is necessary for transmission. The mixing pattern can be modified by assigning workers and equipment to individual barns or groups. Decreasing the movement of people and equipment between groups increases the probability that next contact is with the same animal, reducing the effective contact rate by $33 \%(\beta=0.128)$ and $66 \%(\beta=0.059)$.

(v) Salmonella survives very well outside the gastrointestinal environment. Strict sanitation and disinfection are necessary to reduce contamination of workers and equipment. We tested the effect of improving hygiene by increasing the rate of decontamination by $33 \%(\alpha=0.076)$ and $66 \%$ $(\alpha=0.153)$.

Palliative treatments (e.g. electrolytes) have the potential of decreasing Salmonella-induced mortality without a reduction of the infectious period. In addition to the intervention methods described above, we evaluated the effect of such treatments on the course of an outbreak by reducing the mortality rate by half $(m=0.023)$.

Table II lists different control scenarios and the change in model parameters linked to each intervention scenario. To simulate an ongoing outbreak, control measures were applied at day 20 when the number of infected animals was 5 . The effectiveness of intervention scenarios was ranked based on 3 criteria: (1) predicted prevalence at the endemic equilibrium (deterministic model), (2) the accumulated number of cases until the effective reproduction number $\left(R_{e}\right)$, which is the product between $R_{0}$ and the proportion of susceptible animals and uncontaminated vectors in a given moment during the outbreak, reached 1 


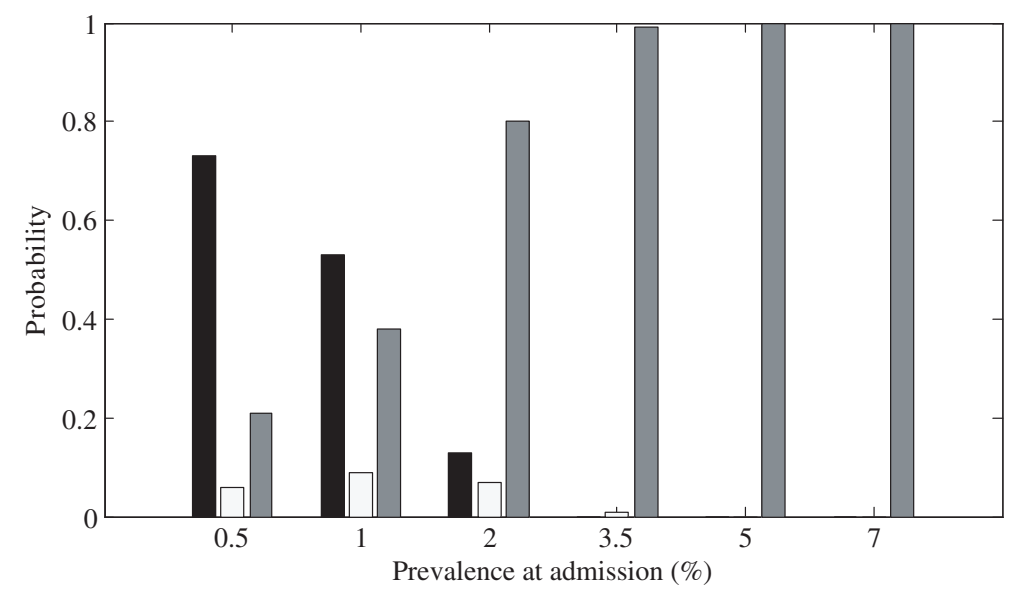

Figure 3. Probability of no transmission events (black bar), transmission, but less than 20 events per year (light grey bar), or more than 20 transmission events per year (dark grey bar) when different prevalence at admission were assumed.

(threshold value at which the outbreak is considered controllable) (deterministic model), and (3) the median number of cases during the outbreak (stochastic model).

\subsection{Intervention strategies to prevent Salmonella outbreaks}

From the list of interventions strategies described in the preceding section, those interventions that are suitable for preventing outbreaks in Salmonella-free farms (i.e., immunization, restricting vector movements between groups, and hygiene measures) were evaluated. For three values of prevalence admission $(1,3.5$, and $7 \%$ ), the intervention strategies were ranked based on the number of simulations in which the introduction of infected animals did not result in a transmission event. For more realistic simulation of outbreak management, the effect of simultaneous application of the most effective intervention strategies was evaluated.

\section{RESULTS}

\subsection{Risk of Salmonella spread following introduction}

We assessed the consequences of introducing infected animals in an operation free of Salmonella. Different degrees of exposure to Salmonella were simulated by varying the
Salmonella prevalence in admitted calves. The median number of infected animals introduced in one year ranged from one animal, when prevalence at admission was $0.5 \%$, to 26 animals when prevalence at admission was $7 \%$. The probability that introduced positive animals did not cause a new case in a year dropped from 0.73 (prevalence of $0.5 \%$ ), to 0.13 (prevalence of $2 \%$ ) (Fig. 3). At prevalence higher than $2 \%$, the probability of having more than 20 new cases a year was close to 1 (Fig. 3). More than one infected animal can be introduced in a single event. As the cluster of introduced index cases increased, the probability of not having an outbreak decreased and the median of the outbreak size and duration increased. The introduction of one positive animal did not result in further new cases in $62 \%$ of the simulations, but introducing three positive animals in one single event resulted in outbreaks in $75 \%$ of the simulations, with a median of 142 cases.

\subsection{Intervention strategies to control ongoing Salmonella outbreaks}

The estimated $R_{0}$ for the case study was 2.4. Figure 4 represents the predicted evolution of $R_{e}$ by the deterministic model for the baseline scenario and the most effective 


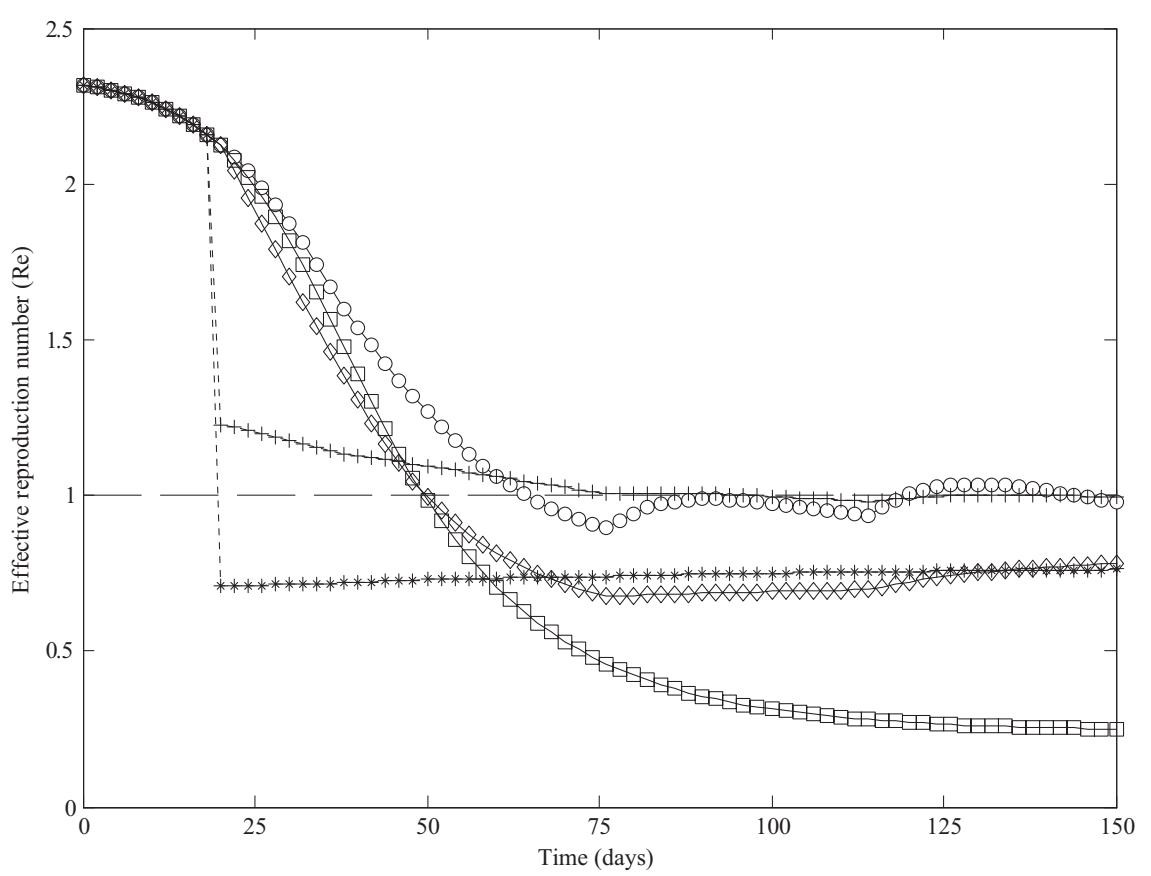

Figure 4. Simulated effective reproductive number $\left(R_{e}\right)$ (deterministic model) for the baseline scenario (o) and the most effective intervention scenarios to control an ongoing outbreak (Adm100 ( $\square$ ), Cont66 (*), $\operatorname{Imm} 75(\diamond)$, Hyg66 (+)).

scenarios outlined in Table II. For the baseline scenarios, $R_{e}$ decreased rapidly from its initial value $\left(R_{0}\right)$ to 1 at day 64 . Afterwards, $R_{e}$ fluctuated around 1 , indicating endemic stage. The fluctuations around one were due to the demographics of the system (i.e., variable admission rate and pulse weaning). The complete closure of the facilities to new admissions (Adm100) had the largest impact on $R_{e}$ (Fig. 4). In the stochastic simulations, Adm100, Imm75, and Cont66 had outbreaks with smaller size and duration compared to other scenarios (Fig. 5). Although, decreasing the environmental survival of Salmonella by $66 \%$ (Hyg66) was not enough to reduce and maintain $R_{e}$ below 1 (Fig. 4), the simulated outbreaks were small, with a median lower than the scenario Imm75 (Fig. 5). Combining Cont66 and Hyg66 measures was more effective than combining Cont66 with Imm75 (Fig. 5). Interventions were ranked based on three model outputs (Tab. III). Prevalence at endemic stage ranked Adm100 and Shed 60, the first and fourth most effective interventions, respectively. Cont66 was ranked as the most effective scenario when the criteria were either the accumulated number of cases when $R_{e}$ reached 1 or the total outbreak size.

A scenario in which the mortality rate was halved was simulated to assess the effect of palliative treatments that reduce mortality, but not morbidity. Decreasing the mortality rate by half increased the median of outbreak size to 278 cases and the duration to 237 days.

\subsection{Intervention strategies to prevent Salmonella outbreaks}

Strategies to prevent Salmonella outbreaks in Salmonella-free farms were evaluated at three levels of admission prevalence (Tab. IV). At the lowest prevalence (1\%), assigning workers and equipment to specific groups was the most effective preventive strategy. When the contact rate was reduced by $66 \%$ (Cont66), the probability of not having a new transmitted case in a year increased from 0.53 

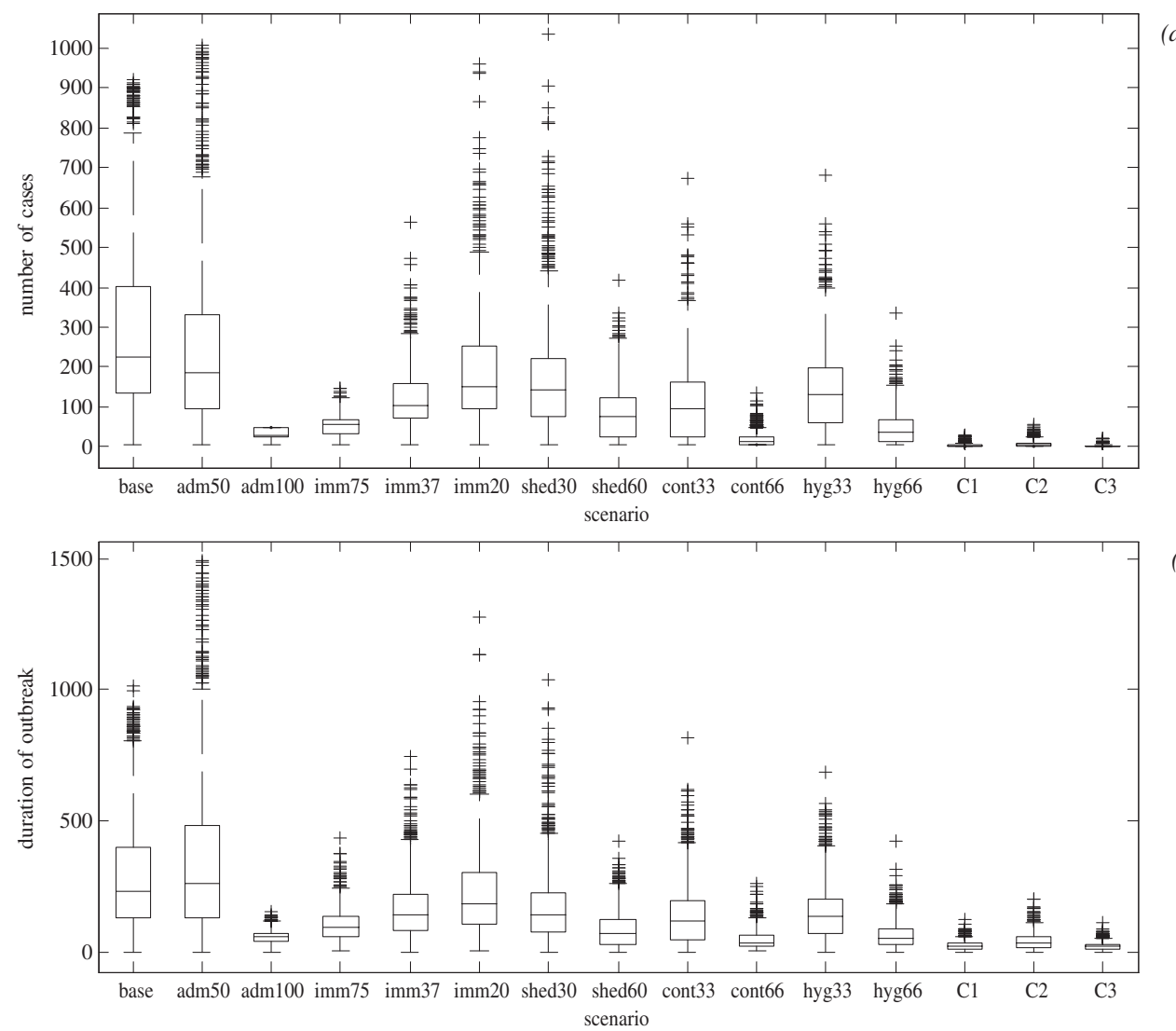

Figure 5. Box plots for the variability in the number of cases during an outbreak (Panel $a$ ) and the duration of the outbreaks (Panel $b$ ) for the baseline scenarios and the scenarios with individual control measures (as listed in Tab. II) and combined strategies (C1: hyg66+cont66, C2: cont66+imm75, C3: hyg66+cont66+imm75). The middle line in the box represents the median, and upper and lower areas of the center box indicate the 75 th and 25 th percentiles.

to 0.80 . The other scenarios showed limited improvement with respect to the base scenario. With admission prevalence of 3.5 and $7 \%$, the Cont66 was also ranked the most effective scenario, but overall the ability of preventing Salmonella cases was very narrow for all the scenarios.

\section{DISCUSSION}

\subsection{Modeling approach}

As dairy farms become larger, the use of off-site calf raising is becoming increasingly common [20]. Biosecurity is an important challenge for calf-raising operations; nearly two-thirds of operations that sent heifers off for rearing sent them to facilities where they had contact with cattle from other operations [18]. In this paper, we reported a mathematical model of Salmonella infection dynamics in a pre-weaned calf population. Previous models of Salmonella transmission in pre-weaned calf populations have focused on closed dairy herds $[13,23]$, and to the best of our knowledge, no transmission models of Salmonella or other enteric pathogens in calfrearing operations have been published. While the structure of the model can be applied to 
Table III. Ranking of the effectiveness of the intervention scenarios to control an ongoing outbreak based on (1) the predicted endemic prevalence (deterministic model), (2) the number of accumulated cases when $R_{e}$ reached the threshold value of 1 (deterministic model) and (3) the median of the number of cases for outbreak (stochastic model).

\begin{tabular}{|c|c|c|c|c|c|c|}
\hline \multirow[t]{2}{*}{ Scenario } & \multicolumn{4}{|c|}{ Deterministic model } & \multicolumn{2}{|c|}{ Stochastic model } \\
\hline & Endemic prevalence & Rank & $N$ cases $\left(R_{e}=1\right)$ & Rank & Total $N$ cases & Rank \\
\hline Base & 10.35 & & 67 & & 226 & \\
\hline Adm50 & 19.37 & 11 & 50 & 6 & 184 & 11 \\
\hline Adm100 & 0 & 1 & 32 & 2 & 27 & 2 \\
\hline Imm75 & 0.36 & 3 & 34 & 3 & 58 & 4 \\
\hline $\operatorname{Imm} 37$ & 4.01 & 5 & 49 & 5 & 105 & 7 \\
\hline $\operatorname{Imm} 20$ & 6.47 & 7 & 57 & 8 & 152 & 10 \\
\hline Shed30 & 6.51 & 8 & 61 & 9 & 145 & 9 \\
\hline Shed60 & 2.89 & 4 & 50 & 7 & 76 & 5 \\
\hline Cont33 & 7.43 & 9 & 65 & 11 & 94 & 6 \\
\hline Cont66 & 0.08 & 2 & 6 & 1 & 12 & 1 \\
\hline Hyg33 & 8.9 & 10 & 61 & 10 & 129 & 8 \\
\hline Hyg66 & 4.78 & 6 & 40 & 4 & 35 & 3 \\
\hline
\end{tabular}

Table IV. Probability of not having transmission events during a year in a farm free of Salmonella for three levels of admission prevalence under different control strategies.

\begin{tabular}{lccc}
\hline Measures & \multicolumn{3}{c}{ Admission prevalence } \\
\cline { 2 - 4 } & $1 \%$ & $3.5 \%$ & $7 \%$ \\
\hline Base & 0.53 & 0 & 0 \\
Imm75 & 0.55 & 0.08 & 0.08 \\
Imm37 & 0.54 & 0.06 & 0.01 \\
Imm20 & 0.54 & 0.03 & 0 \\
Cont33 & 0.62 & 0.10 & 0 \\
Cont66 & 0.80 & 0.46 & 0.20 \\
Hyg33 & 0.54 & 0.03 & 0 \\
Hyg66 & 0.58 & 0.10 & 0 \\
\hline
\end{tabular}

study the control of other enteric pathogens, transmission-related parameters were mostly derived from an outbreak of $S$. Newport, and therefore, they may be applicable to other highly virulent serotypes, such as $S$. Typhimurium, but they may not apply to all Salmonella serotypes.

We adapted the SIR model framework used for indirectly transmitted diseases, such as vector transmitted [1] and nosocomial infections $[2,4]$, to make more explicit the role that personnel and equipment play in the transmission of Salmonella in individually penned calves. Although the indirect transmission was the only route included in the model, direct transmission may occur in individually penned animals if the design of the pens allows animal contact or splashing movements of pathogens between adjacent pens [8]. The host-vector and vector-host transmission terms were both frequency-dependent because we assumed that contact rates between animals and workers and equipment were the result of the daily management activities of the operation, rather than the number of animals or vectors. In addition, both transmission coefficients $\left(\beta_{c}\right.$ and $\left.\beta_{i}\right)$ were set equal based on the assumption that the contact between animal and vector would result on the same 'infective/contamination dose' for both animal and vector. The effect of such assumption on the model outputs was assessed by simulating scenarios having unequal transmission coefficients. With the deterministic approach, the effect of unequal transmission coefficient was minimal for the endemic prevalence ( $<1 \%$ change) and $R_{e}(<0.02$ unit change). For the most probable alternative (i.e. $\beta_{c}>\beta_{i}$ ), with a $\beta_{c}$ two folds greater than $\beta_{i}$, the number of fade-outs predicted by the stochastic model decreased from $60 \%$ to $52 \%$ because the invasion process (i.e., contamination of a vector by the index case) became more effective. 
We used both deterministic and stochastic approaches to address our objectives. Deterministic models facilitate the estimation of parameters and model analysis. In addition, they provide a simple, intuitive measure of control efficacy and risk of infection, $R_{0}$. Although threshold values such as $R_{0}$ are useful in guiding disease control policies, dynamics, stochasticity and finite population effects can also have a major influence on the effectiveness of control strategies $[9,11]$. For that reason, we analyzed the stochastic eventdriven model to assure that the conclusions obtained by the deterministic model held and to provide the size and duration distribution of the outbreaks for such populations.

\subsection{Risk of Salmonella outbreaks upon introduction in calf-raising operations}

Our first objective was to address what the consequences were of introducing Salmonella in a naïve population of unweaned calves. The $R_{0}$ determines the potential of an infectious agent to start an outbreak [1]. In an indirect transmission model, like the one presented here, two generations were required to close the cycle of infection from one calf to another calf, and therefore one calf could infect 5.6 calves $\left(=R_{0}^{2}\right)$, indicating the high potential for starting an outbreak. The distribution of outbreak sizes had a heavy right tail (Fig. 5) indicating that although large outbreaks were more rare events, they were also possible. The high turnover rate of the facilities, which resulted in a continual replenishment of the susceptible pool favored the persistence of the pathogen in the premises.

The risk of Salmonella spread with repeated introductions was evaluated by varying Salmonella prevalence of admitted calves ranging from 0.5 to $7 \%$. High prevalence or clusters of infected calves at admission can be expected when animals come from large number of farm sources, especially if animals are mixed during transportation [21]. With prevalence at admission higher than $2 \%$, the probability of spreading the infection being close to 1 highlighted the need for reducing the risk of introducing Salmonella with incoming calves. The importance of screening admitted animals for Salmonella was also illustrated when the control strategies to prevent Salmonella were evaluated; when the prevalence at admission was $3.5 \%$ or greater, the overall ability of preventing Salmonella cases was very limited for all intervention scenarios (Tab. IV).

\subsection{Assessment of intervention strategies}

Our second objective was to evaluate the effect of intervention strategies to control and prevent Salmonella outbreaks. We presented the ranking of interventions based on different criteria (Tab. III). By ranking the interventions with both deterministic and stochastic approaches, we assessed the consistency and robustness of the ranking. Overall, the median of the total cases for outbreak and the average endemic prevalence ranked interventions similarly, which suggests that both deterministic and stochastic approaches are appropriate to draw qualitative conclusions on effectiveness of control strategies. For all the criteria assessed, Cont66 and Adm100 were either the first or second most effective scenarios; however the rankings were less consistent with the less effective control scenarios (Tab. III). Scenarios differed in their ability to reach and to maintain $R_{e}$ below 1 , as well as how fast they were able to decrease $R_{e}$ compared to the baseline scenario (Fig. 4). The hygiene scenarios were not able to maintain $R_{e}$ below 1 because we chose rather conservative reduction in contamination (33 and 66\%) to acknowledge the difficulties in eliminating Salmonella from farm environments. Persistence of environmental contamination after sanitation has been described for calf units [12]. In a prospective study carried out in 86 broiler houses, the proportion of contaminated houses after cleaning and disinfection was 38.4\% [16]. For the reported case study [6], once the control measures aimed to reduce contamination of the personnel and control traffic flow pattern were in place, the outbreak began to decrease in a few days. The abrupt reduction that both hygiene and movement control had on $R_{e}$ may have helped to control the outbreak (Fig. 4). 
Interventions that decreased the average number of cases for outbreak also decreased the variance of number of cases and duration of the outbreak (Fig. 5). Some intervention scenarios (Cont33 and Adm50) were ranked very disparately. Reducing the admission by $50 \%$ had a low impact on the outbreak size because it had two opposite effects; it decreased the pool of susceptible calves, which in turn caused an increase in the proportion of infected calves. A higher proportion of infected calves increased the force of infection for the vector population. Results indicated that admission of immunized calves $(>75 \%$ of the admissions) could reduce considerable the size and duration of the outbreaks. Previous work examining the protective immunity of current Salmonella vaccines [10] indicated that the vaccine provided only protection against serotypes within the same serogroup of the vaccine strain, and therefore reaching high percentage of immunized animals may become challenging.

The efficacy of the interventions in preventing outbreaks was mostly driven by the number of introduced infected animals. At low prevalence, reducing the movements of workers and equipment was the most effective way to prevent cases. Due to the high turnover of hosts in the system, efforts should be made to prevent introduction of infected calves. In that regard, knowledge of the health status of the farm sources is important to design an effective prevention plan. Controlling traffic flow patterns and assigning workers to specific groups are effective ways to prevent and control outbreaks. Assigning workers to specific groups can be accomplished by either pairing workers with groups of calves or by using a separate set of clothing and boots for each group. When feasible, the complete closure of the facilities should be considered to stop outbreaks.

Acknowledgements. This project has been funded with Federal funds from the National Institute of Allergy and Infectious Diseases, National Institute of Health, Department of Health and Human Services, under Contract No. NO1-AI-30054. We thank Dr Charles Gardner, Dr Roger Ellis and Dr Sonya Kelsey and the other veterinarians and farm employees involved in the field outbreak investigation and data collection from the heifer raising facility.

\section{REFERENCES}

[1] Anderson R.M., May R.M., Infectious diseases of humans: dynamics and control, Oxford University Press, Oxford, 1992.

[2] Austin D.J., Bonten M.J.M., Weinstein R.A., Slaughter S., Anderson R.M., Vancomycin-resistant enterococci in intensive-care hospital settings: Transmission dynamics, persistence, and the impact of infection control programs, Proc. Natl. Acad. Sci. USA (1999) 96:6908-6913.

[3] Bender J.B., Reducing the risk of Salmonella spread and practical control measures in dairy herds, Bovine Pract. (1994) 28:62-64.

[4] Cooper B.S., Medley G.F., Scott G.M., Preliminary analysis of the transmission dynamics of nosocomial infections: stochastic and management effects, J. Hosp. Infect. (1999) 43:131-147.

[5] Fecteau M.-E., House J.K., Kotarski S.F., Tankersley N.S., Ontiveros M.M., Alcantar C.R., Smith B.P., Efficacy of ceftiofur for treatment of experimental salmonellosis in neonatal calves, Am. J. Vet. Res. (2003) 64:918-925.

[6] Gardner C.E., Nydam D.V., Ellis R.G., Kelsey S., McDonough P., Warnick L.D., Case report Management of an outbreak of salmonellosis on a commercial calf raising unit, Bovine Pract. (2004) $38: 147-154$.

[7] Gillespie D.T., General method for numerically simulating stochastic time evolution of coupled chemical-reactions, J. Comput. Phys. (1976) 22:403434 .

[8] Hardman P.M., Wathes C.M., Wray C., Transmission of salmonellae among calves penned individually, Vet. Rec. (1991) 129:327-329.

[9] Heffernan J.M., Smith R.J., Wahl L.M., Perspectives on the basic reproductive ratio, J. R. Soc. Interface (2005) 2:281-293.

[10] House J.K., Ontiveros M.M., Blackmer N.M., Dueger E.L., Fitchhorn J.B., McArthur G.R., Smith B.P., Evaluation of an autogenous Salmonella bacterin and a modified live Salmonella serotype choleraesuis vaccine on a commercial dairy farm, Am. J. Vet. Res. (2001) 62:1897-1902.

[11] Lloyd-Smith J.O., Cross P.C., Briggs C.J., Daugherty M., Getz W.M., Latto J., et al., Should we expect population thresholds for wildlife disease? Trends Ecol. Evol. (2005) 20:511-519. 
[12] McLaren I.M., Wray C., Epidemiology of Salmonella typhimurium infection in calves - Persistence of Salmonellae on calf units, Vet. Rec. (1991) 129:461-462.

[13] Nielsen L.R., van den Borne B., van Schaik G., Salmonella Dublin infection in young dairy calves: Transmission parameters estimated from field data and an SIR-model, Prev. Vet. Med. (2007) 79:46-58.

[14] Rankin J.D., Taylor R.J., The estimation of doses of Salmonella typhimurium suitable for the experimental production of disease in calves, Vet. Rec. (1966) 78:706-707.

[15] Reynolds D.J., Morgan J.H., Chanter N., Jones P.W., Bridger J.C., Debney T.G., Bunch K.J., Microbiology of calf diarrhoea in southern Britain, Vet. Rec. (1986) 119:34-39.

[16] Rose N., Beaudeau F., Drouin P., Toux J.Y., Rose V., Colin P., Risk factors for Salmonella persistence after cleansing and disinfection in French broilerchicken houses, Prev. Vet. Med. (2000) 44:9-20.

[17] Rothenba H., Mortality and morbidity in calves with salmonellosis, J. Am. Vet. Med. Assoc. (1965) 147:1211-1214.

[18] USDA, Off-site heifer raising on U.S. dairy operations, 2007, \#508.1107, USDA:APHIS:VS:CEAH:
National Animal Health Monitoring System, Fort Collins, 2007.

[19] Van den Driessche P., Watmough J., Reproduction numbers and sub-threshold endemic equilibria for compartmental models of disease transmission, Math. Biosci. (2002) 180:29-48.

[20] Wolf C.A., Custom dairy heifer grower industry characteristics and contract terms, J. Dairy Sci. (2003) 86:3016-3022.

[21] Wray C., Todd N., McLaren I.M., Beedell Y.E., The epidemiology of Salmonella in calves - the role of markets and vehicles, Epidemiol. Infect. (1991) 107:521-525.

[22] Wray C., Davies R.H., Salmonella infections in cattle, In: Wray C., Wray A. (Eds.), Salmonella in domestic animals, CABI Publishing, Wallingford, 2000, pp. 169-190.

[23] Xiao Y.N., Bowers R.G., Clancy D., French N.P., Understanding the dynamics of Salmonella infections in dairy herds: a modelling approach, J. Theor. Biol. (2005) 233:159-175.

[24] You Y., Rankin S.C., Aceto H.W., Benson C.E., Toth J.D., Dou Z., Survival of Salmonella enterica serovar Newport in manure and manure-amended soils, Appl. Environ. Microbiol. (2006) 72:5777-5783. 